\title{
Applying plastic surgery principles to ovarian tissue transplantation
}

\author{
Amanda Y. Shen ${ }^{1}$, Warren M. Rozen ${ }^{2}$, Alex Polyakov ${ }^{3,4,5,6}$, Kate Stern ${ }^{3,4,5,6}$, Genia Rozen ${ }^{3,4,5,6}$ \\ ${ }^{1}$ Royal Melbourne Hospital, Melbourne, VIC, Australia; ${ }^{2}$ Department of Plastic and Reconstructive Surgery, Peninsula Clinical School, Central \\ Clinical School, Faculty of Medicine, Monash University, Frankston, Victoria, Australia; ${ }^{3}$ Reproductive Services, Royal Women's Hospital, Parkville, \\ VIC, Australia; ${ }^{4}$ Department of Obstetrics and Gynaecology, Royal Women's Hospital, Parkville, VIC, Australia; ${ }^{5}$ University of Melbourne and \\ Gynaecology Research Centre, Parkville, VIC, Australia; ${ }^{6}$ Melbourne IVF, East Melbourne, VIC, Australia \\ Contributions: (I) Conception and design: WM Rozen, G Rozen; (II) Administrative support: WM Rozen, G Rozen; (III) Provision of study materials \\ or patients: WM Rozen, G Rozen; (IV) Collection and assembly of data: AY Shen, WM Rozen, G Rozen; (V) Data analysis and interpretation: AY \\ Shen, WM Rozen, G Rozen; (VI) Manuscript writing: All authors; (VII) Final approval of manuscript: All authors. \\ Correspondence to: Amanda Y. Shen, MBBS. 300 Grattan Street, Parkville, VIC 3050, Australia. Email: amanda.yang.shen@gmail.com.
}

\begin{abstract}
Ovarian tissue cryopreservation (OTC) and transplantation is an innovative procedure increasingly utilized to help preserve fertility after gonadotoxic treatments especially in cancer patients. Approximately $30 \%$ of autotransplanted patients are able to achieve live birth, typically with the help of in-vitro fertilization. Numerous techniques and grafting sites have been described to continue to increase this figure. In the field of plastic surgery, tissue grafting has been successful performed for thousands of years and knowledge in this area has been significantly refined. A qualitative review of the literature using PubMed, Cochrane, SCOPUS and Medline databases was performed to look for articles relating to ovarian tissue transplantation (OTT) and comparisons made to plastic surgery tissue grafting. Many parallels were found between the principles of grafting in plastic surgery and the principles of OTT, including pre-operative patient optimization, suitable donor site selection, tissue harvest and preparation, graft site choice, immobilization of the graft and post-operative care. Consideration of the benefits and risks of using orthotopic versus heterotopic recipient sites is also highly important with regards to graft take, morbidity and ease of access of oocyte collection. We believe that ongoing discussion between disciplines can have the potential to improve knowledge, surgical techniques and patient outcomes.
\end{abstract}

Keywords: Skin graft; ovarian tissue transplantation (OTT); fertility

Submitted Jan 13, 2021. Accepted for publication Jun 23, 2021.

doi: $10.21037 / g s-21-24$

View this article at: https://dx.doi.org/10.21037/gs-21-24

\section{Introduction}

Increasing long-term survivorship after cancer highlights the fact that the effects of gonadotoxic treatment are becoming increasingly important and the field of oncofertility is rapidly expanding (1). Of all cancers diagnosed in Australia in 2019, 6\% were in adult women under 45 years (2).

Several different options, such as egg and embryo freezing, currently exist for fertility preservation. Ovarian tissue cryopreservation (OTC) provides a uniquely relevant opportunity in that it enables the preservation of many thousands of primordial follicles without ovarian stimulation. It is the only option for prepubertal girls or in patients who have insufficient time to undergo ovarian stimulation to freeze eggs $(1,3-5)$. It is considered an innovative procedure by ESHRE guidelines and there have been over 170 live births achieved worldwide following subsequent ovarian tissue grafting $(6,7)$.

Ovarian cortical tissue containing primordial follicles is typically harvested laparoscopically prior to cancer treatment and cryopreserved. When the patient is medically 
fit, in long-term remission or cured, desires fertility and has been confirmed to have ovarian failure, ovarian tissue transplantation (OTT) or grafting may be undertaken to transplant the ovarian tissue to a well vascularized tissue bed. Common locations include the remaining ovary, broad ligament, ovarian fossa or other (heterotopic) locations such as the abdominal wall $(8,9)$. Depending on the location of the grafting site, the process may allow for spontaneous pregnancy in the future without in vitro fertilisation (IVF), though this is commonly required (3).

It is believed that approximately $30 \%$ of autotransplanted patients achieve live birth with the aid of this procedure and experience with OTT is increasing rapidly (10). Numerous surgical techniques have been documented, with the selected method and graft location dependent on patient circumstances such as the indication for cryopreservation, presence of at least one ovary or previous pelvic radiotherapy (4). While success rates are improving every year, there is still a need to determine the optimal approach regarding factors such as grafting site selection, tissue volumes and other factors which may help reduce post-graft follicle loss and improve chances of spontaneous pregnancy $(4,11)$. It is anticipated that increasing numbers of women will be returning for ovarian tissue grafting, further demonstrating the importance of the exploration of these factors and further research in this area $(8,12)$.

Cross-disciplinary collaborations synthesising expertise to develop new approaches to address complex problems have become an increasingly important part of medicine. Plastic surgery techniques have previously been applied in gynaecological procedures, predominantly using the upper rungs of the reconstructive ladder, including vulval surgery, congenital vaginal agenesis and fistula repairs (13). The aim of this article is to explore the opportunity for plastic surgery principles, in particular those relating to tissue grafting, to be applied to the procedure of OTT.

\section{Methods}

An electronic literature review was conducted using the PubMed, Cochrane, SCOPUS and Medline databases for publications dated from 1988-2018 for all relevant full text articles published in English, to evaluate surgical techniques for ovarian tissue autotransplantation (AT). Initial searches using the following $\mathrm{MeSH}$ terms were used: ovarian tissue cryopreservation AND ovarian tissue auto-transplantation; additional keyword searches were performed using "ovarian tissue transplantation", "ovary transplantation", "ovarian implantation", "ovary implantation", "ovarian tissue allografting", "ovarian auto-transplantation", "ovary allografting". Articles abstracts and/or full article details were then reviewed by two reviewers (GR, WMR) in order to determine article suitability.

The published and peer-reviewed full-text articles identified from this search were evaluated by reviewing the titles and abstracts. Only the full-text articles which reviewed or studied OTC and AT techniques which aided in identifying gaps in our current knowledge were included. In addition, the bibliographies of the included articles were reviewed for further studies. Two reviewers (GR, WMR) independently reviewed the included reports. Data from the text, graphs, and tables were qualitatively analysed.

Inclusion criteria were articles related to OTT. NonEnglish studies were excluded. Qualitative information was sought regarding OTT as well as principles of tissue grafting as used in plastic surgery to look for areas of similarity and overlap. Table 1 summarizes some of the similarities in findings, which we will discuss in more detail in the following text.

\section{Patient factors affecting graft take}

Patient factors are highly important when considering graft take. Most women undergo OTT after a history of cancer (commonly sarcoma, gynaecological and breast cancer and Hodgkin disease), though some do so after benign disease (14). Treatment follows successful oncological therapy and in women who would like children and have experienced ovarian failure as a result of their cancer treatment (14). Numerous AT studies have compared factors such as age, previous exposure to chemotherapy or radiotherapy and amount of pelvic radiotherapy received (10). So far, no specific evidence has been found regarding the effect of patient comorbidities such as diabetes or smoking on OTT success.

OTT should generally be performed at a maximum age of 45 years, after discussion with the patient's oncologist and consideration of oncological prognosis $(14,15)$. Previous studies have shown good success rates in women who had tissue frozen below the age of 30 , while those above 35 years of age at time of freezing have very low rates of spontaneous pregnancy (10). Those who have had pelvic radiation will also have compromised blood supply to the area, and it must be considered whether pregnancy is safe for these patients due to the risk of adhesions causing difficult access, friable vasculature and possibility of increased risk of 
Table 1 Comparison of skin grafting and ovarian tissue grafting factors

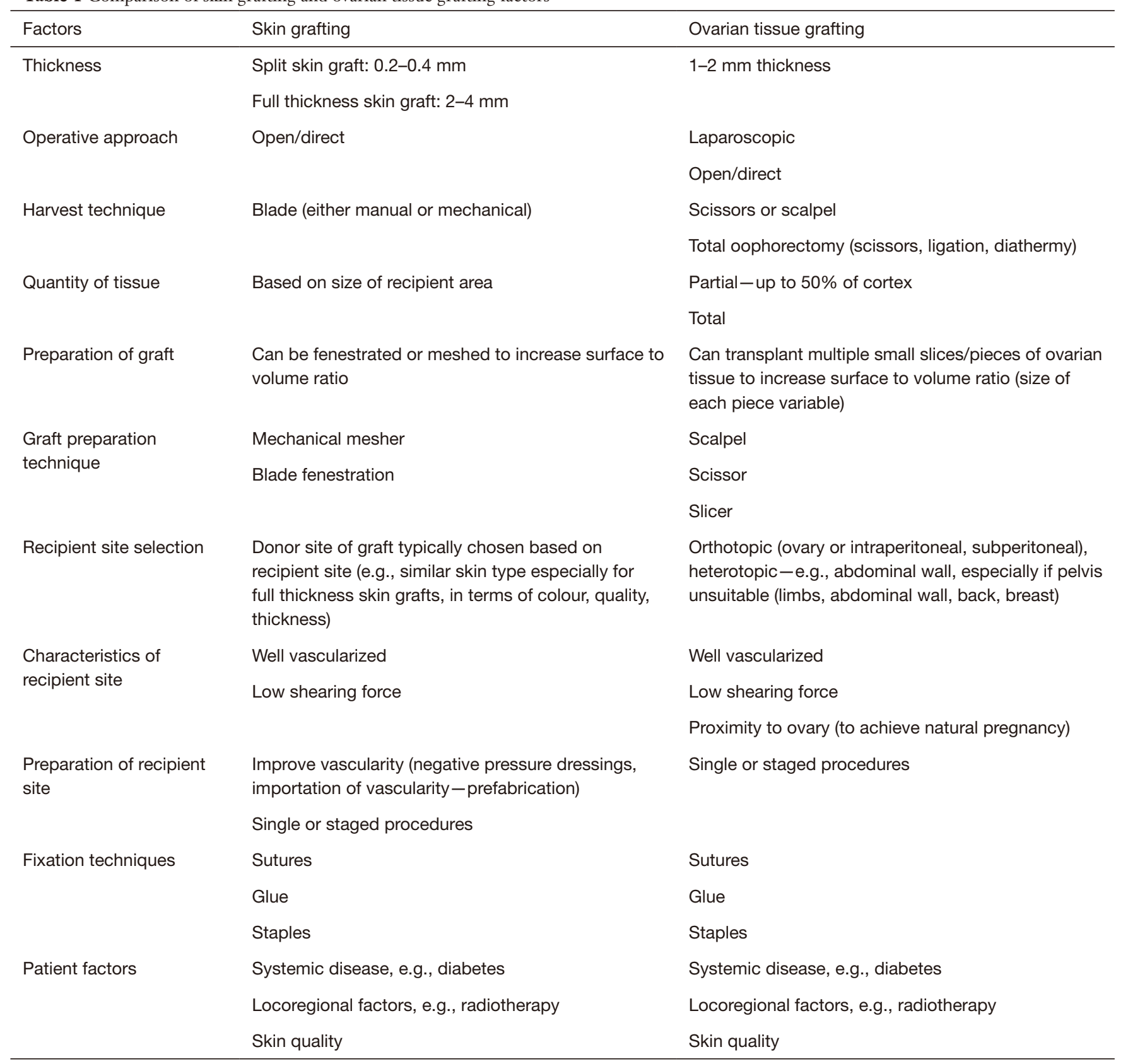

obstetric complications due to uterine irradiation $(10,16)$. A gestational carrier may be required to carry the pregnancy due to irreversible uterine damage (16). There is also a low return rate for patients wanting OTT who have completed cancer treatment, which may be related to a multiple factors (10).

Similarly, when considering patient suitability for skin graft or flap coverage in plastic surgery, it is important to consider age and co-morbidities, especially regarding the quality of the tissues that will be grafted (17). For example, diabetic patients have been shown to have a longer healing time of almost 2 weeks and over five times increased risk of post-operative complications after split-thickness skin graft compared to non-diabetic patients (18). However, most patients do well with skin grafting provided the local factors of bleeding, infection and mobility are addressed. 


\section{Tissue preparation}

Primordial follicles are located just beneath the ovarian surface in the cortical region, and there are several steps in the preparation of the ovarian biopsies before cryopreservation. Firstly, the depth and volume of tissue taken is established. The recommended thickness of ovarian cortex taken is usually $1-2 \mathrm{~mm}(19-21)$, which is in between the thickness of a split-thickness and full-thickness skin graft $(0.2-0.4 \mathrm{~mm}$ thick and 2-4 $\mathrm{mm}$, respectively). Unlike skin grafts, no benefit was seen from thinning the strips further and they in fact had greater risk of follicle loss (22). Generally, the surface-to-volume ratio of the ovarian graft should be high to improve chance of penetration and removal of cryoprotectant agents, reduce risk of ice crystal damage from the freezing and thawing process and to be able to survive the initial hypoxic period during grafting (22).

The removal of the medulla is a crucial step to improve cryoprotectant infiltration into the ovarian cortex during the freezing process. An in vitro experiment comparing the stromal and follicular viability for three decortication methods, including scratching with scalpel blade, cutting with microsurgical scissors and separation with slicer, concluded that blade decortication is the optimal technique for maintaining acceptable follicular conditions without inducing serious stromal impairment (23). Herraiz et al. observed that slicer and microsurgical scissors induced an inhibition in the Hippo pathway, ending in massive follicular activation or burnout (23).

In-vivo animal model studies, however, do not show a difference in terms of follicular density after xenotransplantation regardless of decortication technique (23). In addition to the type of ovarian tissue preparation, there are several other factors such as transport, freezing media, as well as the inevitable posttransplantation hypoxia that may affect outcomes, although many studies appear to use a similar slow-freezing technique for cryopreservation and there is some evidence that the follicular survival rate is similar in ovarian tissue transported on ice for up to 6 hours and in tissue collected and frozen locally $(24,25)$. Some studies have also trialled the use of antiapoptotic drugs in transport and freezing media (24). These have shown some benefit, especially in initial testing, but do not necessarily translate to improved graft success or follicular health after transplantation (26).

In skin grafting, once the desired thickness and quantity of tissue is harvested, the skin can be kept moist in a gauze soaked with saline and can be kept in the refrigerator for up to several weeks and used again on the patient if required. In full thickness skin grafts, excess fat should be trimmed off the dermis due to its poor blood supply and inevitable necrosis and to improve contact with the wound bed. The grafts are typically fenestrated (unless very small) and sometimes meshed to the desired ratio when large grafts are required, as the meshing enables the skin to expand to cover a wider surface area.

\section{Quantity of ovarian tissue transplanted}

The quantity of ovarian tissue transplanted usually depends in part on the amount cryopreserved. Only a part of the tissue is grafted in some cases to preserve opportunity for further procedures, as most papers advise transplanting less than a third to a half each time $(15,27,28)$. A metaanalysis by Pacheco et al. in 2017 found that approximately $34 \%$ of the equivalent surface area of one ovary was transplanted on average, with some women requiring more than one procedure (11). A recent study on the impact of chemotherapy on OTT showed that a follicular density of $\geq 0.3$ follicles $/ \mathrm{mm}^{2}$ (which was the median follicular density of patients not exposed to chemotherapy) and increased number of fragments transplanted ( $\geq 16$ fragments) were associated with an increased probability of ovarian functional recovery (27).

A highly variable volume and thickness of grafts has been used throughout the literature for other bone and soft tissue grafting applications. Skin graft quantity for example, can be classified by the amount of dermis that is included within the graft; a portion (split-thickness) or entire dermis (fullthickness) (29). A large volume of tissue with no vascularised interface is not useful-the less dermis included in the graft, the more likely the chance of graft survival due to a shorter distance needed for oxygenated blood and nutrients to travel in order to perfuse into the graft (29). However, negatives of a thinner skin graft include increased contracture during the healing process and higher risk of damage from shearing or trauma. Like skin grafting, there are several factors that can be optimised in relation to graft quantity in order to increase chance of successful ovarian tissue function post transplantation, namely the density of follicular tissue within the sample, the surface area-to-volume ratio and the number of tissue fragments transplanted.

Using numerous small pieces of ovarian tissue 1-3 mm in size is a technique to improve take, but these tissues left freely floating in a peritoneal pocket or in a pocket below 
the cortical surface of the existing ovary may easily shear away from the graft bed as the patient becomes more active after transplantation. As with any graft, shearing forces disrupt neovascularization between graft tissue and graft bed, which reduces graft survival. A study by Meirow and colleagues indirectly discussed this concept by removing cortical tissue from both ovaries in a single patient (20). During grafting, strips of ovarian tissue were positioned in the left ovary and small pieces of tissue were placed in the right. The large pieces of tissue were firmly fixed to the underlying surface in subcortical pockets, whereas the small ones were deposited free floating in subcortical pockets. Follicular activity was observed only in the left ovary and pregnancy eventually achieved (20). These findings suggest that the size of cortical tissue pieces should be large enough to be placed flat on the underlying surface and prevent movement of the tissue when the woman is mobilising postoperatively.

\section{Site selection}

Two graft site principles are utilised for OTT; orthotopic (tissue is returned to the original site-the ovary or its surrounds within the pelvis) or heterotopic (to alternative sites 'outside the normal place'). Exact definitions of the specific locations for transplantation in these two methods vary slightly in the literature (30). Tissue may be also transplanted into orthotopic and heterotopic sites simultaneously (31). It is important to consider the recipient site from the perspective of rate of graft success, oocyte collection access and vulnerability to trauma.

Orthotopic transplantation, where fragments are grafted onto the remaining ovary or into a peritoneal pocket created in the ovarian fossa, has been shown to be significantly more successful than heterotopic methods. Some studies have found that out of 130 live births reported in their sample population, only two were from heterotopic transplantation (32-34). There are a number of reasons why orthotopic grafts may be more successful-these include vascularisation, a more favourable environment in terms of temperature, pressure and paracrine factors which are important for graft take and oocyte activity, and location close to the Fallopian tube which may increase chance of spontaneous conception (22).

Heterotopic transplantation can be used in cases where the pelvis is unsuitable for transplantation, for example due to radiation, adhesions or a separate pathology (e.g., endometriosis) (33). Transplantation into the anterior abdominal wall and a submuscular pocket on the lateral pelvic wall (which may also be alternatively classified as orthotopic) has led to successful live births $(33,34)$. There are also case series where restoration of ovarian function has occurred following heterotopic transplantation between the rectus muscle and rectus sheath and subcutaneously in both the lower abdomen and forearm (35-37). While heterotopic sites have the advantage of being easier to access and having a higher capacity for grafts, assisted reproduction is required for pregnancy (37).

Like skin grafts, the blood supply to the graft is highly dependent on the recipient graft bed and this is an important consideration when choosing the graft type and technique. In non-abdominal sites, dermis, muscle and fascia have the richest blood supply while fat and subcutaneous tissue tend to be less vascular (38). Dermal ovarian grafts may be impractical due to the superficial nature of the layer which leads to increased risk of injury or shearing forces, making intramuscular grafts likely to be the best option. The mobility of the site should also be considered; limbs will tend to be much more mobile than the trunk or abdomen and any grafts will be subject to more forces.

\section{Characteristics of graft site}

An optimum graft site needs to achieve a balance between a well vascularised bed with adequate haemostasis to allow revascularisation whilst preventing haematoma or fluid formation under the graft (39). If both ovaries are absent, fragments may be transplanted into a peritoneal window. To try and induce neoangiogenesis and revascularisation through granulation tissue, a two-step technique may be utilised, with the initial step to make a peritoneal window performed 7 days before tissue transplantation (32). However, this requires two procedures and is not generally performed. The principle of the 'delay phenomenon' is also widely accepted in plastic surgery, where an initial surgical intervention promotes ischaemic conditions in the tissue, leading to increased neovascularisation for the purpose of flap designs (40).

In skin grafting, haematoma formation is the most common cause of skin graft failure as the clot prevents contact between graft and the recipient bed and hence prevents revascularisation of the graft (41). The risk of haematoma under a skin graft can be reduced by adequate haemostasis and the use of tie-over dressings (29). In orthotopic transplantation, different techniques have been 
utilised to ensure the ovarian cortical pieces are exposed to the medulla and its vascular network when transplanted. The ovary may be denuded to reveal the medulla by removing a large piece of cortex, or subcortical pockets are made through various forms of incisions to feed the fragments into $(20,42)$. Like with skin grafts, haematoma reduces the contact between the grafted ovarian fragments and the graft bed where diffusion of nutrients need to occur, and may increase risk of graft failure. One technique used to reduce the risk of haematoma formation is microcautery with pulsatile irrigation with heparinized saline (9). However, caution must be taken to minimise cauterisation so as not to impair revascularisation.

One of the limitations of both skin and ovarian transplantation is the ischaemic time immediately after transplantation before revascularisation occurs. Skin grafts are avascular and rely on the graft bed to provide perfusion via imbibition, which typically occurs after the first 24 hours and continues the process of graft take over the next 5-7 days (39). Similarly, ovarian tissue fragments can sit in a hypoxic environment with nutrients provided via diffusion for approximately $3-5$ days but up to 10 days before full revascularization (43). During this time, the grafted tissue undergoes ischaemic reperfusion injury, leading to follicle loss; methods aimed at reducing this hypoxic period have shown improved primordial follicle survival (43).

\section{Surgical techniques}

There are a number of different documented surgical approaches for transplanting cryopreserved ovarian tissue. As previously discussed, the choice between orthotopic or heterotopic graft sites depend on patient disease factors as well as any adjuvant therapies such as removal of ovaries or pelvic irradiation. Various surgical techniques such as robotic surgery and use of allografts such as Alloderm have also been trialled in an attempt to increase the success of follicular stimulation from OTT, but ongoing experimentation is likely to be required (44).

A graft needs to be securely fixed to its recipient bed to reduce bleeding, infection and shearing forces and promote neoangiogenesis to allow the graft to take (45). Heterotopic OTT in superficial sites may be especially prone to graft movement but it is also important when fixing tissue within the peritoneal cavity. Fixation of tissue in OTT varies significantly, depending on graft location and surgeon preference. Transplantation of tissue fragments onto a denuded ovary have been attached using sutures (such as 7/0 or $8 / 0$ polypropylene or $9 / 0$ nylon), intercede and/or fibrin glue. They may also be placed in either subcortical pockets in the ovary or peritoneal pockets, with the incisions closed if required with either fibrin glue, staples or sutures (e.g., $4 / 0$ vicryl) to hold them in place $(4,46)$. It should be noted, however, that these interventions minimize graft mobility but may increase risk of haematoma or seroma formation due to impaired drainage, and tissues should be handled as little as possible to prevent mechanical damage, especially when fragments are small (4).

Skin grafts are also immobilised using various techniques, including sutures, staples or fibrin glue, influenced by location of the graft site, age of the patient and indication for the graft. Different dressings are also utilised to minimise shearing forces, with simple foam and staples to more complex tie-over dressings (39). The main difference is that skin grafts are superficial and can often overlie an area of high mobility, e.g., muscle, and patients are often required to rest in bed for a number of days to allow for maximum chance of graft take. OTT patients may also benefit from counselling of rest or minimal activity for a period of time to reduce graft tissue movement.

\section{Transplant outcomes}

Ovarian transplantation outcomes have been studied in depth and have shown varying degree of success. A recent review by Sheshpari et al. demonstrated that ovarian function can return in 50-95\% of transplant recipients and occurs generally between 3-6 months; maintenance of function lasted for approximately $9-84$ months (47). The reported overall clinical pregnancy rate after transplantation was $58 \%$, with $65 \%$ of fresh tissue and $45 \%$ of frozen tissue transplant recipients achieving live birth (47). The result of assisted reproduction techniques after grafting is still lower than that in the general population undergoing IVF and this may be linked to the low ovarian reserve resulting from the follicle loss likely due to atresia observed at each stage during cryopreservation and after transplantation, although approximately $80 \%$ of follicles lost are during the final postgrafting stage $(7,43)$. Adipose tissue-derived stem cells have been used as a potential method to improve vascularisation and oxygenation in order to reduce ischaemic injury to the follicular reserve (24).

When considering transplant outcomes is also important to discuss the possibility of transplanting tumour cells. Several studies have assessed the risk of reintroducing malignant tissue during ovarian transplantation, with the 
highest risk associated with haematological malignancies such as leukaemia and ovarian cancers, including nonprimary ovarian deposits (14). Similarly, in plastic surgery, skin grafts, flaps and other reconstructive options are carefully selected from healthy skin as free as possible from sun damage or previous trauma to prevent grafting malignant or pre-malignant skin changes onto the recipient site.

\section{Conclusions}

The core principles in tissue grafting appear to have parallels across different specialties, whether it be grafting of skin or ovarian tissue. While graft take is multifactorial, several strategies have been described to improve the quality of grafted ovarian tissue and the clinical outcomes of OTT. As in skin grafting, there needs to be good quality and quantity of ovarian tissue harvest, an appropriate recipient site with a well vascularized graft bed, strong graft fixation technique to prevent shearing of neovasculature and optimization of comorbidities such as diabetes or immunosuppression. We believe that ongoing discussion between disciplines can have the potential to improve knowledge, surgical techniques and patient outcomes.

\section{Acknowledgments}

Funding: None.

\section{Footnote}

Peer Review File: Available at https://dx.doi.org/10.21037/ gs-21-24

Conflicts of Interest: All authors have completed the ICMJE uniform disclosure form (available at https://dx.doi. org/10.21037/gs-21-24). WMR serves as an unpaid editorial board member of Gland Surgery from Mar 2018 to Feb 2023. The other authors have no conflicts of interest to declare.

Ethical Statement: The authors are accountable for all aspects of the work in ensuring that questions related to the accuracy of integrity of any part of the work are appropriately investigated and resolved.

Open Access Statement: This is an Open Access article distributed in accordance with the Creative Commons
Attribution-NonCommercial-NoDerivs 4.0 International License (CC BY-NC-ND 4.0), which permits the noncommercial replication and distribution of the article with the strict proviso that no changes or edits are made and the original work is properly cited (including links to both the formal publication through the relevant DOI and the license). See: https://creativecommons.org/licenses/by-nc-nd/4.0/.

\section{References}

1. Shapira M, Dolmans MM, Silber S, et al. Evaluation of ovarian tissue transplantation: results from three clinical centers. Fertil Steril 2020;114:388-97.

2. Australian Institute of Health and Welfare. Cancer in Australia 2019. Available online: https://www.aihw.gov.au/ reports/cancer/cancer-in-australia-2019/data

3. Oktay K, Karlikaya G. Ovarian function after transplantation of frozen, banked autologous ovarian tissue. N Engl J Med 2000;342:1919.

4. Dolmans MM, Manavella DD. Recent advances in fertility preservation. J Obstet Gynaecol Res 2019;45:266-79.

5. Levine JM, Kelvin JF, Quinn GP, et al. Infertility in reproductive-age female cancer survivors. Cancer 2015;121:1532-9.

6. Martinez F. Update on fertility preservation from the Barcelona International Society for Fertility PreservationESHRE-ASRM 2015 expert meeting: indications, results and future perspectives. Hum Reprod 2017;32:1802-11.

7. Andersen ST, Pors SE, Poulsen LC, et al. Ovarian stimulation and assisted reproductive technology outcomes in women transplanted with cryopreserved ovarian tissue: a systematic review. Fertil Steril 2019;112:908-21.

8. Andersen CY. Success and challenges in fertility preservation after ovarian tissue grafting. Lancet 2015;385:1947-8.

9. Silber S. Ovarian tissue cryopreservation and transplantation: scientific implications. J Assist Reprod Genet 2016;33:1595-603.

10. Macklon KT. Cryopreservation of ovarian tissue works, but challenges remain. Fertil Steril 2020;114:281-2.

11. Pacheco F, Oktay K. Current Success and Efficiency of Autologous Ovarian Transplantation: A Meta-Analysis. Reprod Sci 2017;24:1111-20.

12. Rozen G, Agresta F, Gook D, et al. Success and challenges in fertility preservation after ovarian tissue grafting. Lancet 2015;385:1947.

13. Nicholson R, Shafi MI. Plastic surgical techniques in gynaecology. Obstet Gynaecol Reprod Med 
2013;23:253-5.

14. Lotz L, Dittrich R, Hoffmann I, et al. Ovarian Tissue Transplantation: Experience From Germany and Worldwide Efficacy. Clin Med Insights Reprod Health 2019;13:1179558119867357.

15. Beckmann MW, Lotz L, Toth B, et al. Concept Paper on the Technique of Cryopreservation, Removal and Transplantation of Ovarian Tissue for Fertility Preservation. Geburtshilfe Frauenheilkd 2019;79:53-62.

16. Rozen G, Rogers P, Chander S, et al. Clinical summary guide: reproduction in women with previous abdominopelvic radiotherapy or total body irradiation. Hum Reprod Open 2020;2020:hoaa045.

17. Boyce DE, Shokrollahi K. Reconstructive surgery. BMJ 2006;332:710-2.

18. Ramanujam CL, Han D, Fowler S, et al. Impact of diabetes and comorbidities on split-thickness skin grafts for foot wounds. J Am Podiatr Med Assoc 2013;103:223-32.

19. Silber SJ, DeRosa M, Goldsmith S, et al. Cryopreservation and transplantation of ovarian tissue: results from one center in the USA. J Assist Reprod Genet 2018;35:2205-13.

20. Meirow D, Levron J, Eldar-Geva T, et al. Pregnancy after transplantation of cryopreserved ovarian tissue in a patient with ovarian failure after chemotherapy. $\mathrm{N}$ Engl J Med 2005;353:318-21.

21. Sonmezer M, Oktay K. Fertility preservation in female patients. Hum Reprod Update 2004;10:251-66.

22. Donfack NJ, Alves KA, Araújo VR, et al. Expectations and limitations of ovarian tissue transplantation. Zygote 2017;25:391-403.

23. Herraiz S, Monzó S, Gómez-Giménez B, et al. Optimizing ovarian tissue quality before cryopreservation: comparing outcomes of three decortication methods on stromal and follicular viability. Fertil Steril 2020;113:609-617.e3.

24. Nisolle M, Brichant G, Henry L. Optimizing the outcomes of ovarian tissue transplantation. Fertil Steril 2020;113:547-8.

25. Ladanyi C, Mor A, Christianson MS, et al. Recent advances in the field of ovarian tissue cryopreservation and opportunities for research. J Assist Reprod Genet 2017;34:709-22.

26. Henry L, Fransolet M, Labied S, et al. Supplementation of transport and freezing media with anti-apoptotic drugs improves ovarian cortex survival. J Ovarian Res 2016;9:4.

27. Poirot C, Fortin A, Lacorte JM, et al. Impact of cancer chemotherapy before ovarian cortex cryopreservation on ovarian tissue transplantation. Hum Reprod 2019;34:1083-94.

28. Gellert SE, Pors SE, Kristensen SG, et al. Transplantation of frozen-thawed ovarian tissue: an update on worldwide activity published in peer-reviewed papers and on the Danish cohort. J Assist Reprod Genet 2018;35:561-70.

29. Shimizu R, Kishi K. Skin graft. Plast Surg Int 2012;2012:563493.

30. Demeestere I, Simon P, Emiliani S, et al. Orthotopic and heterotopic ovarian tissue transplantation. Hum Reprod Update 2009;15:649-65.

31. Rosendahl M, Loft A, Byskov AG, et al. Biochemical pregnancy after fertilization of an oocyte aspirated from a heterotopic autotransplant of cryopreserved ovarian tissue: case report. Hum Reprod 2006;21:2006-9.

32. Donnez J, Dolmans MM, Demylle D, et al. Livebirth after orthotopic transplantation of cryopreserved ovarian tissue. Lancet 2004;364:1405-10.

33. Tammiste T, Kask K, Padrik P, et al. A case report and follow-up of the first live birth after heterotopic transplantation of cryopreserved ovarian tissue in Eastern Europe. BMC Womens Health 2019;19:65.

34. Stern CJ, Gook D, Hale LG, et al. Delivery of twins following heterotopic grafting of frozen-thawed ovarian tissue. Hum Reprod 2014;29:1828.

35. Chen C. Pregnancy after human oocyte cryopreservation. Lancet 1986;1:884-6.

36. Kim SS. Assessment of long term endocrine function after transplantation of frozen-thawed human ovarian tissue to the heterotopic site: 10 year longitudinal follow-up study. J Assist Reprod Genet 2012;29:489-93.

37. Oktay K, Economos K, Kan M, et al. Endocrine function and oocyte retrieval after autologous transplantation of ovarian cortical strips to the forearm. JAMA 2001;286:1490-3.

38. Saladin KS. Anatomy and Physiology: The Unity of Form and Function, 8th Edition. McGraw-Hill Education; 2017.

39. Thornton JF, Gosman AA. Skin Grafts and Skin Substitutes and Principles of Flaps. Selected Readings in Plastic Surgery 2004;10:1.

40. Milton SH. The effects of "delay" on the survival of experimental pedicled skin flaps. Br J Plast Surg 1969;22:244-52.

41. Flowers RS. Unexpected postoperative problems in skin grafting. Surg Clin North Am 1970;50:439-56.

42. Donnez J, Dolmans MM, Pellicer A, et al. Restoration of ovarian activity and pregnancy after transplantation of cryopreserved ovarian tissue: a review of 60 cases of 
reimplantation. Fertil Steril 2013;99:1503-13.

43. Roness H, Meirow D. FERTILITY PRESERVATION: Follicle reserve loss in ovarian tissue transplantation. Reproduction 2019;158:F35-44.

44. Oktay K, Taylan E, Kawahara T, et al. Robot-assisted orthotopic and heterotopic ovarian tissue transplantation techniques: surgical advances since our first success in 2000. Fertil Steril 2019;111:604-6.

45. Burleson R, Eiseman B. Nature of the bond between partial-thickness skin and wound granulations. Surgery 1972;72:315-22.

Cite this article as: Shen AY, Rozen WM, Polyakov A, Stern K, Rozen G. Applying plastic surgery principles to ovarian tissue transplantation. Gland Surg 2021;10(7):2266-2274. doi: 10.21037/ gs-21-24
46. Oktay K, Bedoschi G, Pacheco F, et al. First pregnancies, live birth, and in vitro fertilization outcomes after transplantation of frozen-banked ovarian tissue with a human extracellular matrix scaffold using robot-assisted minimally invasive surgery. Am J Obstet Gynecol 2016;214:94.e1-9.

47. Sheshpari S, Shahnazi M, Mobarak H, et al. Ovarian function and reproductive outcome after ovarian tissue transplantation: a systematic review. J Transl Med 2019;17:396. 